\title{
BAYESIAN FACTOR ANALYSIS
}

by

Gordon M. Kaufman*

and

S. James Press $\frac{*}{*}$

May 1973

$662-73$

Not to be quoted without the authors' permission.

${ }^{*}$ Gordom M. Kaufman, M.I.T. Sloan School of Management

$* *$ S. J. Press, University of Chicago, Graduate School of Business and the Department of Economics

This Working Paper also appeared in the University of Chicago series as Report 7322, Center for Mathematical Studies in Business and Economics, Department of Economics and Graduate School of Business, April 1973. 


\section{Bayes1an Factor Analysis}

by

G. M. Kaufman

Massachusetts Institute

of Technology

\section{Introduction}

In this paper we discuss the problem of factor analysis from the Bayesian viewpoint. First, the classical factor analysis model is generalized in several directions. Then, prior distributions are adopted for the parameters of the generalized model and posterfor distributions are developed in the light of observed data. Finally, we develop a large sample marginal posterior distribution for the elements of the factor loading matrix. It w11l be seen that the Bayesian approach provides a formal mechanism for using subjective prior information to eliminate ambiguities and dogmatic constraints ever present In earlier factor analysis models.

There has been a large literature devoted to the classical factor analysis problem (see, for example, [7] Mulaik, 1972 and his 183 references). However, though subjective information has been brought to bear on many earlier analyses in an Informal and often ad hoc way, a formal Bayes approach was not suggested unt11 recently (See 18] Press, 1972, p. 317). That approach, however, was limited by the constraints of the classical factor analysis model. Below, we will show how generalizing the form of the classical podel permits an operationally useful Bayesian solution to be generated.

On leave at the Departments of Administrative Sciences and Statistics, Yale University, 1972-1973. 
Let $\underline{x}_{1}, \ldots, \underline{x}_{n}$ denote independent and identically distributed observations of the random $m$-vector $\underline{X}$, and suppose

$$
\frac{x}{(m \times 1)}=\frac{\mu}{(m \times 1)}+\frac{\Lambda}{(m \times s)} \cdot \frac{f}{(s \times 1)}+\frac{\varepsilon}{(m \times 1)}
$$

where $\mathscr{L}(\underline{f})=\mathrm{N}(\underline{0}, \underline{I})$ and $\mathscr{L}(\underline{\varepsilon})=\mathrm{N}(\underline{0}, \underline{\Sigma}), \underline{\Sigma}>\underline{0}$

( $\Sigma$ is a positive definite symetric matrix); that is, the probability law of $f$ is an s-variate normal distribution with zero mear. and Identity covariance matrix, and the probability law of $\underline{\varepsilon}$ is a normal distribution with mean zero and positive definite covariance matrix $\underline{\Sigma}$. Suppose $\underline{f}$ and $\underline{\varepsilon}$ are independent. The s-vector $\underline{f}$ is often called the vector of common factors, and with $\operatorname{var}(\underline{f})=\underline{I}$, the varfous factors are postulated to be mutually orthogonal; we will treat only the orthogonal factor model here since the extension of these results to an oblique factor model is conceptually straightforward. $\Lambda$ is often referred to as the factor loading matrix and it is assumed to be non-random; its rows represent $m$ sets of weights to be assigned to the $s$ factors. $\varepsilon$ (called the disturbance or error vector) denotes a vector which includes both the effects of factors specific to each of the components of $\underline{X}$, and also, terms which are intended to account for non-1inearities and measurement errors in the model. The expected value of $\underline{X}$ is denoted by ‥ All terms on the right hand side of (1.1) are assumed to be unobservable.

\subsection{Classical Mode1}

The classical model of factor analysis presupposes that 
(a) E is diagonal, and

(b) $\mathrm{s} \leq \mathrm{m}$.

The explanation of (a) is that any correlation observed among the components of $\underline{X}$ is accounted for by the common factors (so that the elements of $\underline{\varepsilon}$ are uncorrelated). The implication of (b) is that there are no more factors than there are observed attributes (and usually, 8 is assumed to be considerably smaller than $m$ ). The first problem of classical factor analysis is to specify $s$ and to estimate $\Lambda$ and the diagonal elements of $\underline{\Sigma}$. Then, checks on the goodness of fit of the model must be made, and it is sometimes also of interest to estimate the factors themselves.

\subsection{Bayesian Model}

The Bayesian model of factor analysis developed below assumes the following:

(c) $\Sigma$ is a general positive definite symetric covarlance matrix,

(d) $s \geqslant m$,

(e) $\operatorname{rank}(\underline{\Lambda})=\mathrm{m}$.

The factor analysis model with assumptions (c) - (e) will be seen to be more general and flextble than the classical model. The cost of greater generality and flexibllity is that we will be required to assess informative prior distributions for a large set of parameters. A broad comparison of the two approaches is given below. 


\subsection{Comparison of Classical and Bayesian Models}

Adoption of the Bayesian viewpoint has a major advantage: It allows explicit introduction of subjective judgment about model structure in a way that copes neatly with specification error and with the 1dentification problem that characterizes factor analytic models. (A precise account ensues below.) The arbitrary and often dogmatic assumptions commonly introduced in order to obtain unique estimators of model parameters are replaced with multivarlate prior distributions of high dimensionality which can express all degrees of subjective views ranging from dogmatic assumptions regarding the parameters to complete vagueness. All of the usual problems that arlse when encoding subjective judgments about many fointly dependent uncertain quantities are present: What constitutes a class of flexible yet analytically tractable prior distributions? How does one elicit a particular member of this class in practice? And so on. Each of the escape routes commonly employed in the literature--Jeffreys -like diffuse priors, natural confugate priors-- suffers from one or more fatal defects, as will be seen in the next section, and so, we w11l be forced to consider more complicated functional forms for priors.

The Bayesian model has been posited with more underlying common factors than observable attributes (In diametric opposition to the classical approach). In almost any real world situation we take only a small fraction of the total number of possible measurements. That is, 
there are generally a great many factors underlylng any real object or construct but only a few observations are taken on each object.

If we grant that $s \geqslant m$ in the real world, how then is parsimony to be achieved? That 18, how will it be posstble to conclude our analysis with a representation of the observed data in a lower dimensional space, a common obfective of factor analysis? The answer lies in our cholce of the prior distribution. Partition the factor loading matrix and the factor vector as shown below (where $t$ denotes transpose) :

$$
\begin{aligned}
& \left.\underset{(m \times s)}{\Lambda}=\underset{(\operatorname{mxp})}{\left(\underline{\Lambda}_{1},\right.} \underset{(\operatorname{mx}(8-p))}{\underline{\Lambda}_{2}}\right), \\
& \left.\underset{(s \times 1)}{\underline{f}^{t}}=\underset{(p \times 1)}{\left(\underline{f}_{1}^{t},\right.} \underset{((s-p) \times 1)}{\underline{f}_{2}^{t}}\right), p<s .
\end{aligned}
$$

Suppose we belleve a priorl that $p$ factors are really adequate to explain the obeerved data, $p<8$. (In sow problews it may be deasrable to take $p<m$. Th1s is not a general requirement, however.) This subjective bellef can be translated into a formal prior distribution by assuming that a prtort, $\Lambda_{2}$ Iles in a small nelghborhood of the sero atrix. With this partitioning, (1:1) becomes

$$
\underline{X}=\underline{\mu}+\underline{\Lambda}_{1} \underline{f}_{1}+\underline{\Lambda}_{2} \underline{f}_{2}+\underline{\varepsilon} .
$$


By adjusting the hyperparameters (the parameters of the prior distribution) appropriately, the effect of the $\left(\underline{A}_{2} \underline{f}_{2}\right)$ term in the posterior distribution can be made as insignificant as is deemed desirable. However, one never really knows with certainty that exactly p factors "explain" the $\underline{X}^{\prime} s$. A more common state of affairs is that one has a strong (subjective) belief that among $s$ candidates for factors, $p$ of them account for a large portion of the variance of the $\underline{X}^{\prime} s$. Thus, in the Bayesian model there is considerable flexibility regarding the choice of number of factors and how Important they are relative to one another.

In the Bayeslan model, by permitting components of $\underline{\varepsilon}$ to be correlated we account for the possibility of specification error; that is, omission of one or more factors from the model. By contrast, the classical model assumes all components of $\underline{\varepsilon}$ are uncorrelated (assumption (a)).

Finally, it will be seen that the rotation problem of classical factor analysis can be completely circumvented by implementing the Bayesian analysis described below. That is, if subjectlve information is introduced through the class of priors suggested, the usual rotational identification problem will disappear 80 that all parameters of the factor loading matrix w11l be identified, and at least in large samples, it will be straightforward to make posterior inferences about the loadings since the appropriate distribution will turn out to be normal (truncated). 
The problem of parameter Identification is discussed in the next section. Then in Section 3, we develop the main result of the paper, namely, the pusterior distribution of the factor loading matrix and obtain a modal point estimator. In Section 4, it is explained how Informative prior information may be brought to bear on the problem of assessing a particular member of the appropriate fam1ly of prior distributions.

\section{Identification}

Let $\underline{\bar{x}} \equiv n^{-1} \sum_{1}^{n} \underline{x}_{f}$ and $\underline{s} \equiv \sum_{1}^{n}\left(\underline{x}_{f}-\underline{\bar{x}}\right)\left(\underline{x}_{f}-\underline{\bar{x}}\right)^{t}$ denote the sample mean and covariance matrices, respectively. Since $\mathcal{L}(\underline{X})=N(\underline{\mu}, \underline{\Omega})$, where $\left(\omega_{1 j}\right) \equiv \underline{\Omega} \equiv \underline{\Lambda}^{t}+\underline{\Sigma},(S, N)$ is sufficlent for $\underline{(\Lambda, \Sigma)}, N \equiv \mathfrak{n}-1$, (we are not generally interested in making inferences about $\underline{\mu}$ ). Thus, the likelihood function is given by

$$
\ell(\underline{\Lambda}, \underline{\Sigma} \mid \underline{s}, N)=\left|\underline{\Lambda}^{t}+\underline{\Sigma}\right|^{-N / 2} \exp \left[(-1 / 2) \operatorname{tr}\left(\underline{\Lambda}^{t}+\underline{\Sigma}\right)^{-1} \underline{s}\right]
$$

In the sequel we shall assume that $N \geqslant m$ so that $\underline{S}>\underline{0}$ with probability one. Note that the likelihood function may be written in the equivalent form

$$
e^{*}(\underline{\Lambda}, \underline{\Omega} \mid \underline{\underline{S}}, N)=|\underline{\Omega}|^{-\mathrm{N} / 2} \exp \left[(-1 / 2) \operatorname{tr} \underline{\Omega}^{-1} \underline{\underline{S}}\right],
$$


where $\ell^{*}$ denotes a function with $m(m+1) / 2$ functionally Independent components $\omega_{1 j}$ and $l^{*}$ is constant over al1 $\Lambda$ and $\underline{\Sigma}$ such that $\underline{\Lambda}^{t}+\underline{\Sigma}=\underline{\Omega}$. Since $\underline{\Omega}$ is composed of $m(m+1) / 2$ functionally independent elements and $\left(\Lambda^{t}+\underline{\Sigma}\right)$ is composed of $[m s+m(m+1) / 2]$ functionally independent elements we are faced with an identification problem akin to that that arises in the analysis of simultaneous equation systems ${ }^{1}$ and Drèze's commentary thereupon appliles with equal force- here:

A common feature of all these studies is that the a priori restrictions are imposed in exact form, through equalities or inequalities with known, nonstochastic coefficients. Typically, however, the a priori Information provided by economic theory, past observation (casual or systematic), etc., is not of such an exact nature and would be better reflected In probabilistic statements. The weakness of the traditional approach is thus twofold in my opinion: (1) restrictions are imposed in exact form, whereas they should rather take a stochastic form, reflecting the imprecise nature of the prior information; (11) certain parameters are left completely unrestricted, about which some prior information is avallable. ... In other words, the traditional approach lacks flexiblilty in that it relles upon too few a priorl restrictions that are, however, too strict. A more realistic formulation would call for submitting more -eventually all -- parameters to be estimated to stochastic a priori restrictions.

The Bayesion counterpert of the 1dentifleation problin we face is this: Let $A$ be a mesurable aubset of Euclidean ms-space; then the probablilty that the random matrix $\Lambda \in A$, conditional on $\Omega$, remains the same no matter how much data generated according to (1.1) is observed. ${ }^{2}$ Namely, given a set

1 cf. Drèze [1], and Fisher [2].

2 Drèze [1] calls attention to this feature of the problem of inference about parameters of a simultaneous equation. 
of observations generated according to (1.1) and leading to $(\underline{S}, \mathbb{N})$, and given that we have assigned a prior density $f^{\prime}(\underline{\Lambda}, \underline{s})$ jointly to $\Lambda$ and $\Omega$, the conditional (prior) density $p(\underline{\Lambda} \underline{\Omega})$ of $\Lambda$ given $\underline{\Omega}$ is unmodified by $(\underline{S}, N)$. This is almost self-evident upon factoring $f^{\prime}(\underline{\Lambda}, \underline{\Omega})$ Into $\mathrm{p}(\underline{\Lambda} \mid \underline{\Omega}) \mathrm{k}(\underline{\Omega})$ where $\mathrm{k}(\underline{\Omega})$ is the marginal prior density of $\Omega$, and then observing that the likelihood function (2.1) bears on $\Omega$ alone, as in (2.2), whereupon Bayes Theorem Implies that the foint posterior density of $\Lambda$ and $\Omega$ is proportional to $p(\underline{\Lambda \mid \Omega}) \times k(\underline{\Omega}) e^{*}(\underline{\Omega} \mid \underline{S}, N)$.

However, the assertion that $p(\underline{\Lambda} \mid \underline{\Omega})$ remains unaffected by observation of data leading to $(\underline{S}, N)$ must not be taken as Implying that the data is uninformative about $\Lambda$. As $N \rightarrow \infty, \frac{1}{N} S$ converges in probability to the true value, say $\Omega^{\circ}$, of $\underline{\Omega}$, and the posterior marginal density of $\Lambda$ converges (pointwise) to $p\left(\Lambda \mid \Omega^{\circ}\right)$. In this sense, as more and more data accumulates the Bayesian procedure picks out with Increasing probabilistic accuracy that nember of the set $\{p(\underline{\Omega}|\underline{\Omega}| \Omega>0\}$ of conditional prior densities of $\Lambda$. given $\Omega$ corresponding to the true underlying value $\underline{\Omega}^{\circ}$ of $\underline{Q}$.

The marginal density of $\Lambda$ posterfor to observing $(\underline{S}, N)$ will in general differ from the marginal prior density of $\Lambda$, and may do so In such a way as to permit identification of a unique modal value of $\Lambda$ a posteriori, thus resolving the identification problem in a certain sense. The a priori restrictions commonly imposed when doing maximum likelihood estimation of $\Lambda$ are very dogmatic and yet leave us faced with a non-unique estimator of $\Lambda$, one invariant with respect to postmultiplication by an orthogonal matrix. The Bayesian solution is more appealing in principle, but more difficult to implement, demanding 
careful coherent formulation of a large number of stochastic a priori restrictions which may vary in nature from problem to problem.

\section{Prior to Posterior Analysis}

\subsection{Non-informative Priors}

Our approach to analysis of the model (1.1) with assumptions (c), (d), and (e) is to consider various classes of distributions as candidates for priors, and then to develop posterlors by means of Bayes theorem. A natural starting point is to consider the class of non-informative (vague) prior densities ${ }^{1}$ of the form

$$
D^{\prime}(\underline{\Lambda}, \underline{\Sigma}) \cdot|\underline{\Sigma}|^{\alpha-\frac{\mathrm{m}+1}{2}}
$$

Priors of this form are Invarlant with respect to various groups of transformations (depending on the value of $\alpha$ ) and have been used extensively in the Bayesian literature (the case of $\alpha=0$ corresponds

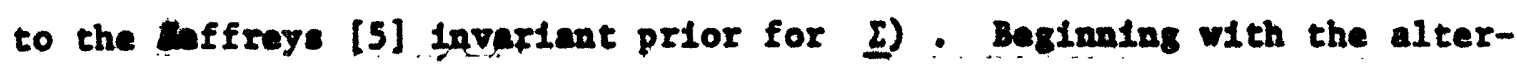
nate parameterization $\Lambda$ and $\underline{\Omega}$, a non-informative (marginal) density for $\underline{\Omega} 1$ s of the same functional form as that of $\underline{\Sigma}$; however, $\Lambda$ given I 18 restricted to be such that $\underline{\Omega}-\underline{\Lambda \Lambda}^{t}>\underline{0}$, so that assignment of a foint prior density

1 Primes will denote prior densities and double primes will denote posterior densities. The symbol will denote proportionality. 


$$
h^{\prime}(\underline{\Lambda}, \underline{\Omega}) \times|\underline{\Omega}|^{\alpha-\frac{\underline{q}+1}{2}}
$$

for $\underline{Q}-\underline{\Lambda}^{t}>\underline{0}$, and equal to zero otherwise implies that

$$
D^{\prime}(\underline{\Lambda}, \underline{\underline{\varepsilon}}) \times\left|\underline{\Lambda \Lambda}^{t}+\underline{\Sigma}\right|^{\frac{m+1}{2}}
$$

In place of (3.1). While compelling in many contexts such priors are now shown to be unsatisfactory here.

Bayes theorem requires that if $\vec{D}(\underline{\Lambda}, \underline{\Sigma})$ denotes the posterior density of $(\underline{\Lambda}, \underline{\Sigma})$,

$$
D^{\prime \prime}(\underline{\Lambda}, \underline{\Sigma}) \propto D^{\prime}(\underline{\Lambda}, \underline{\Sigma}) \ell(\underline{\Lambda}, \underline{\Sigma} \mid \underline{s}, N)
$$

Substituting from (2.1) and (3.1) gives

$$
D^{\prime \prime}(\underline{\Lambda}, \underline{\Sigma}) \propto \frac{\exp \left\{(-1 / 2) \operatorname{tr}\left(\underline{\Lambda} \Lambda^{t}+\underline{\Sigma}\right)^{-1} \underline{s}\right\}}{|\underline{\Sigma}|^{(m+1) / 2}\left|\underline{\Lambda \Lambda}{ }^{t}+\underline{\Sigma}\right|^{N / 2}},
$$

where

$$
D^{\prime \prime}(\underline{\Lambda}) \propto \int_{\underline{\Sigma}>\underline{0}} D^{\prime \prime}(\underline{\Lambda}, \underline{\Sigma}) d \underline{\Sigma}
$$

denotes the marginal posterior density of $\Lambda$. Noting that since $D^{\prime \prime}(-\underline{\Lambda})=D^{\prime \prime}(\underline{\Lambda})$, if $E(\underline{\Lambda} \mid \underline{S})$ exists at al1 we must have $E(\underline{\Lambda} \mid \underline{S})=\underline{0}$;

$1 D^{\prime \prime}(\Lambda)$ is proper only for $\alpha>\frac{m-1}{2}$ or $\alpha<\frac{N-m+1}{2}$. 
that 1s, there are on the average no underlying factors which "explain" the varfance in $\underline{X}$. The same argument holds for a prior of the form $h^{\prime}(\underline{\Lambda}, \underline{\Omega})$. This is an unsatisfactory situation and results from our having selected a class of prior distributions which was not rich enough to accomnodate the demands of our problem. The broader class considered below w11 in fact lead to a satisfactory result.

\subsection{Informative Priors}

Suppose prior information 18 introduced in the form of a jolnt density on $(\underline{\Lambda}, \underline{\Omega})$, Instead of on $(\underline{\Lambda}, \underline{\Sigma})$. Properties of a foint prior density for $(\underline{\Lambda}, \underline{\Omega})$ of the form

$$
\begin{aligned}
& h^{\prime}(\underline{\Lambda}, \underline{\Omega}) \propto \exp \left\{-(1 / 2) \operatorname{tr}\left(\underline{\Lambda}-\underline{\Lambda}_{2}\right) \underline{\nabla}_{2}\left(\underline{\Lambda}-\underline{\Lambda}_{2}\right)^{t} \underline{G}\right\} \\
& \times|\underline{\underline{Q}}|^{-(v+w+1) / 2} \exp \left\{-(1 / 2) \operatorname{tr} \underline{Q}^{-1} \underline{\underline{L}}(\underline{\Lambda})\right\}
\end{aligned}
$$

for $\underline{\Omega}-\underline{\Lambda \Lambda}^{t}>\underline{0}$, and equal to zero otherwlse, w11l now be explored. Here $\Psi(\underline{\Lambda})$ denotes the quadratic form $\underline{S}_{0}+\left(\underline{\Lambda}-\underline{\Lambda}_{1}\right) \underline{V}_{1}\left(\underline{\Lambda}-\underline{\Lambda}_{1}\right)^{t}$. Factoring

$$
h^{\prime}(\underline{\Lambda}, \underline{\Omega}) \propto h_{1}^{\prime}(\underline{\Omega}) h_{2}^{\prime}(\underline{\Lambda} \mid \underline{\Omega})
$$

$h_{2}^{\prime}(\underline{\Lambda} \mid \underline{\Omega})$ is seen to be a truncated normel density, and $\Lambda$ is restricted. to lie in $\left\{\underline{\Lambda:} \underline{\Omega}-\underline{\Lambda \Lambda}^{t}>\underline{0}\right\}$. Factoring 


$$
h^{\prime}(\underline{\Lambda}, \underline{\Omega}) \propto h_{1}^{\prime}(\underline{\Omega} \mid \underline{\Lambda}) h_{2}^{\prime}(\underline{\Lambda})
$$

$h_{1}^{\prime}(\underline{\Omega} \mid \underline{\Lambda})$ is seen to be a truncated Inverted Wishart density, and $\underline{\Omega}$ is reatricted to $11 \mathrm{in}\left\{\underline{\Omega}: \underline{\Omega}-\underline{\Lambda}^{t}>\underline{0}\right\}$.

For very large samples it is intuitively reasonable to replace $\underline{\Omega}$ In $h_{2}^{\prime}(\underline{\underline{\Lambda}} \underline{\Omega})$ with $\frac{1}{N} \underline{S}$; since $\frac{1}{N} \underline{S}$ converges in probabillty to $\underline{\Omega}^{0}$, the true underlying value of $\underline{\Omega}$, and to regard $h_{1}^{\prime}(\underline{\Omega})$ as concentrated In a very saall neighborhood of $\frac{1}{N} \underline{S}$. Upon doing so, $h_{2}^{\prime}(\underline{\Lambda} \mid \underline{\Omega})$ is approxtentely proportional to

$$
\exp \left\{-(1 / 2) \operatorname{tr}\left[\underline{G}\left(\underline{\Lambda}-\underline{\Delta}_{2}\right) \underline{\nabla}_{2}\left(\underline{\Lambda}-\underline{\Lambda}_{2}\right)^{t}+\operatorname{NS}^{-1} \Psi(\underline{\Lambda})\right]\right\}
$$

which upon completing the square in rows (or in colume) of $\Lambda$ can be seen to be a normal density concentrated on $\left\{\underline{\Lambda}: \frac{1}{N} \underline{s}-\underline{\Lambda}^{t}>\underline{0}\right\}$; that 18 , a truncated normal density. The ensuing development renders this Intultive argunat precise, showing that the last expression is also the form of the asymptotic marginal posterior density of $\Lambda$ as $N+\infty$. The most striking feature of this density is that it allows the possibility of a unlque mode, and in so doing offers an a posterlori estimator that resolves the rotational problem. 
How suppose thet e prtort 이스 follows an inverted wishart distribution (truncated, since $\underline{\Omega}$ is bounded below by $\underline{\Lambda}^{t}$ ). Specifically, adopt the conditional prior density

$$
h_{1}^{\prime}(\underline{\Omega} \mid \underline{\Lambda})=\frac{c(\underline{\Lambda})}{|\underline{\Omega}|^{(\nu+m+1) / 2} \exp \left\{(-1 / 2) \operatorname{tr}\left[\underline{\Omega}^{-1} \underline{\phi}(\Lambda)\right]\right\}}
$$

for $v>m-1$ and $\psi(\Lambda)>\underline{0}$, where $\underline{\varphi(\Lambda)}$ is defined in $(3.3), c(\underline{\Lambda})$ denotes the proportionality constant (which depends on $\Lambda$ ) rendering $h_{1}^{\prime}(\underline{\Omega} \mid \underline{\Lambda)}$ a proper density, and where $(\underline{\Lambda}, \underline{\Omega})$ is restricted to lie in $\left\{(\underline{\Lambda}, \underline{\Omega}): \underline{\Omega}>\underline{0}, \underline{\Omega}-\underline{\Lambda \Lambda}^{t}>\underline{0}\right\}$. Note that $\left\{\underline{\Lambda}_{1}, \underline{s}_{0}, \underline{v}_{1}, \nu\right\}$ is a set of hyperparameters which must be assessed on the basis of prior information. (cf. Section 4 for methods of assessment). The hyperparameters satisfy the constraints: $\underline{s}_{0}>0, \underline{v}_{1}>\underline{0}, v>0$.

The constant $c(\Lambda)$ is evaluated as follows. Since

$$
\begin{aligned}
& \int_{\Omega-\Lambda \Lambda} h_{>0}^{\prime}(\Omega \mid \Lambda) d \Omega=1, \\
& {\left[c(\underline{\Lambda)}]^{-1}=\int_{\underline{\Omega}-\underline{\Lambda}^{t}>0} \frac{\exp \left\{(-1 / 2) \operatorname{tr}\left[\Omega^{-1} \underline{\psi}(\Lambda)\right]\right\}}{|\Omega|(\nu+m+1) / 2} d \Omega .\right.}
\end{aligned}
$$


Transforming from $\underline{\Omega}$ to $\underline{H}=\underline{\Omega}^{-1}$, with Jacobian $\mathrm{J}(\underline{\Omega}+\underline{H})=|\underline{H}|^{-(\mathrm{m}+1)}$, gives

$$
[c(\underline{\Lambda})]^{-1}=\int_{\left(\Lambda \Lambda^{t}\right)^{-1}-\underline{H}>\underline{0}} \mid(\nu-m-1) / 2 \exp \{(-1 / 2) \operatorname{tr}[\underline{H} \psi(\underline{\Lambda})]\} d \underline{H} .
$$

Now transforming from $H$ to $\underline{p}=(1 / 2)[\underline{\Psi}(\underline{\Lambda})]^{1 / 2} \underline{H}[\underline{\Psi}(\Lambda)]^{1 / 2}$, with

$$
\begin{aligned}
& \text { Jacobian }{ }^{1} \mathrm{~J}(\underline{H} \rightarrow \underline{\mathrm{P}})=|\underline{\psi}(\underline{\Lambda})|^{-(\mathrm{m}+1) / 2} \text {, gives } \\
& \left.[c(\underline{\Lambda})]^{-1}=\mid \underline{\Psi} \underline{\Lambda}\right)\left.\right|^{-\nu / 2} \int_{\underline{K}-\underline{P}>\underline{0}}|P|^{(\nu-m-1) / 2} e^{-t r} \underline{P} d \underline{P},
\end{aligned}
$$

where $\underline{K} \equiv(1 / 2)[\psi(\underline{\Lambda})]^{1 / 2}\left(\underline{\Lambda \Lambda}^{t}\right)^{-1}[\Psi(\underline{\Lambda})]^{1 / 2}$. Note that up to a scale constant, the last integral is the nultivariate analogue of the incomplete gamma function. From Herz [3], p. 488, we may express the last Integral as a confluent hypergeometric function of matrix argument. Thus,

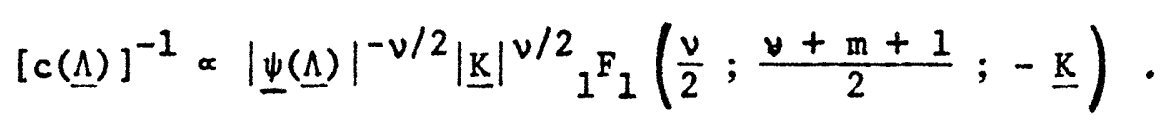

Recalling that the hypergeometric functions are symetric functions of their arguments, and using the definition of $\underline{K}$, gives

$$
\left.\left.[c(\Lambda)]^{-1} \propto|\underline{\Lambda}|^{t}\right|_{1} ^{-\nu / 2} F_{1}\left(\frac{\nu}{2} ; \frac{\nu+m+1}{2}-(1 / 2)\left(\Lambda \Lambda^{t}\right)^{-1} \underline{\psi} \underline{\underline{L}}\right)\right)
$$

$1[\psi(\Lambda)]^{1 / 2}$, and all other matrix square roots in the sequel, are assumed to be positive definite symmetric matrices. 
Combining (3.4) and (3.5) gives the joint prior density

$$
h^{\prime}(\Lambda, \underline{\Omega}) \propto \frac{\hat{h}_{2}^{\prime}(\Lambda)\left|\Lambda \Lambda^{t}\right|^{v / 2} \exp \left\{(-1 / 2) t r \underline{\Omega}^{-1} \Psi(\Lambda)\right\}}{|\underline{\Omega}|^{(\nu+m+1) / 2}{ }_{1} F_{1}\left(\frac{v}{2} ; \frac{\nu+m+1}{2} ;(-1 / 2)\left(\Lambda \Lambda^{t}\right)^{-1} \Psi(\Lambda)\right)} .
$$

Substituting (2.2) and (3.6) into Bayes theorem ((3.2) with $\Sigma$ replaced by $\Omega$ ) gives the foint posterior density

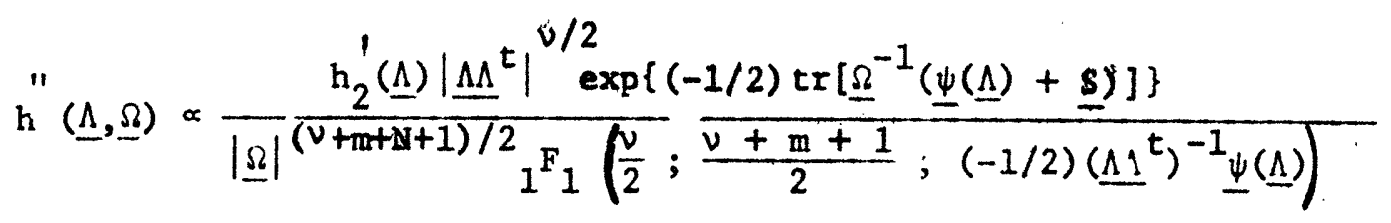

when $\underline{\Omega}-\underline{\Lambda}^{t}>\underline{0} ; h^{\prime \prime}(\underline{\Lambda}, \underline{\Omega})$ is zero in the region where $\underline{\Omega}-\underline{\Lambda \Lambda}^{t}$. is not positive definite. The marginal posterior density of the factor loading matrix is given by

$$
h^{\prime \prime}\left(\underline{\Lambda)} \equiv \int_{\underline{\Omega}-\underline{\Lambda \Lambda}} h^{\prime \prime}(\underline{\Lambda}, \underline{\Omega}) \mathrm{d} \underline{\underline{0}} .\right.
$$

Substituting (3.7) into (3.8) gives

$$
h^{\prime \prime}(\Lambda) \propto \frac{h_{2}^{\prime}(\Lambda)\left|\Lambda^{t}\right|^{v / 2} I(\Lambda)}{\left.{ }_{1} F_{1}\left(\frac{v}{2} ; \frac{v+m+1}{2} ;(-1 / 2)(\underline{M})^{t}-1 \underline{\Psi} \underline{1}\right)\right)}
$$


where

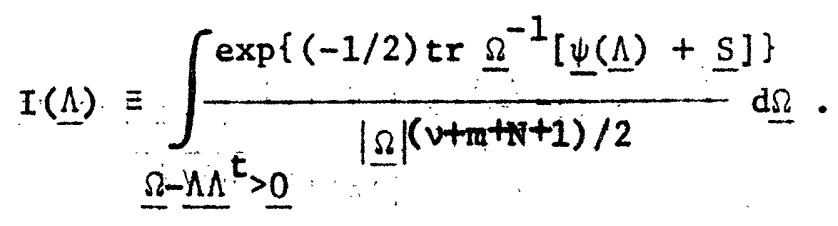

Using the same argument used to evaluate $c(\Lambda)$ above, we find

$$
I(\Lambda) \propto \frac{1^{F} 1\left(\frac{v+N}{2} ; \frac{v+N+m+1}{2} ;-(1 / 2)\left(\Lambda^{t}\right)^{-1}[\Psi(\Lambda)+\underline{s}]\right)}{\left|\Lambda^{t}\right|^{(\nu+N) / 2}} .
$$

Substituting (3.10) into (3.9) gives

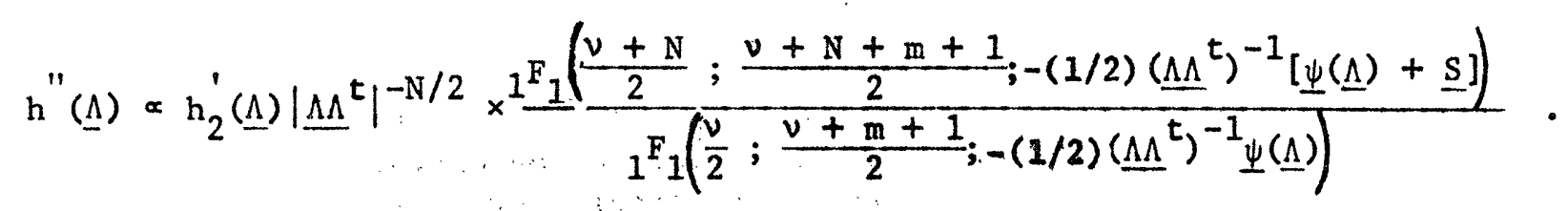

The next problem is to select the class of marginal prior densities on $\Lambda$ so that the class is not only sufficiently rich to accomodate diverse types of prior information, but also, it ylelds a proper and statistically meaningful posterior density, consistent with analytical tractibility. We suggest the class with hyperparameters $\left.\underline{A}_{1}, \underline{A}_{2}, \underline{V}_{1}, \underline{V}_{2} \underline{S}_{0}, \underline{G}, v\right\}$ $\underline{v}_{2}>\underline{0}, \underline{G}>\underline{0}$,

$$
\begin{aligned}
& h_{2}^{\prime}(\Lambda) \propto|\underline{\Lambda \Lambda}|^{t}{ }^{-\nu / 2} F_{1}\left(\frac{\nu}{2} ; \frac{\nu+m+1}{2} ;-(1 / 2)\left(\Lambda \Lambda^{t}\right)^{-1} \underline{\psi}(\Lambda)\right) \\
& \times \exp \left\{(-1 / 2) \operatorname{tr}\left[\underline{G}\left(\Lambda-\underline{A}_{2}\right) \underline{v}_{2}\left(\Lambda-\underline{A}_{2}\right)^{t}\right]\right\},
\end{aligned}
$$

which upon being combined with $h_{1}^{\prime}(\underline{\Omega} \mid \underline{\Lambda})$ as displayed in (3.4) gives a joint prior of the form (3.3). 
Substituting (3.12) into (3.11) generates the class of marginal posterior densities

$$
\begin{aligned}
h^{\prime \prime}(\Lambda) & \propto \frac{\underline{1}_{1}\left(\frac{\nu+N}{2} ; \frac{\nu+N+m+1}{2} ;-1 / 2\left(\underline{\Lambda} \underline{L}^{t}\right)^{-1}[\underline{\Psi}(\underline{\Lambda})+\underline{S}]\right)}{|\underline{\Lambda}|^{t}(\underline{v+N}) / 2} \\
& \times \exp \left\{(-1 / 2) \operatorname{tr}\left[\underline{G}\left(\underline{\Lambda}-\underline{A}_{2}\right) \underline{V}_{2}\left(\underline{\Lambda}-\underline{A}_{2}\right)^{t}\right]\right\} .
\end{aligned}
$$

The result in (3.13) can now be evaluated numerically for given hyperparameters and a given data covariance matrix $\underline{S}$. The task is not simple, however. For example, the ${ }_{1} F_{1}$ function might be expressed In a zonal polynomial series expansion (see, e.g., James [4], p. 477). However, as James pointed out in [4], p. 497:

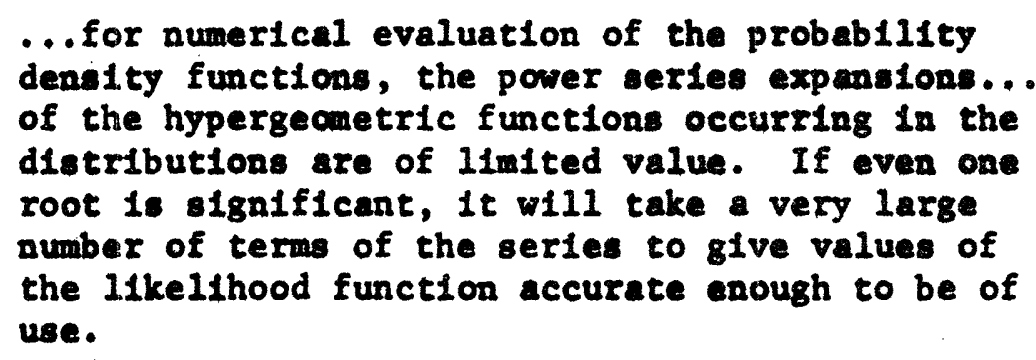

Alternatively, we shall assume we have a moderately large data set

and shall seek a large sample approximation to the result in (3.13).

\subsection{Asymptotics}

Define

$$
J_{N}^{*}(\underline{\Lambda}) \equiv \int_{\underline{K}_{N}-\underline{P}>\underline{0}}|\underline{P}|^{(\nu+N-m-1) / 2} e^{-\operatorname{tr}(P)} d \underline{P},
$$


where $\left.\left.\left.\underline{K}_{\mathrm{N}} \equiv 1 / 2[\underline{\Psi} \underline{\Lambda})+\underline{S}\right]^{1 / 2}(\underline{\Lambda})^{t}\right)^{-1}[\underline{\Psi} \underline{\Lambda})+\underline{S}\right]^{1 / 2}$. From the analysis and evaluation of $c(\underline{\Lambda})$ in Section 3.2 it may be inferred that

$\frac{\left.1_{1}{ }_{1}\left(\frac{\nu+N}{2} ; \frac{\nu+N+m+1}{2} ;-1 / 2(\underline{\Lambda})^{t}\right)^{-1}[\underline{\Psi}(\underline{\Lambda})+\underline{s}]\right)}{\left|\underline{\Lambda} \Lambda^{t}\right|^{(\nu+N) / 2}} \propto \frac{J_{N}^{*}(\Lambda)}{|\underline{s}+\underline{\Psi}(\Lambda)|^{(\nu+N) / 2}}$.

It 1 s shown in Appendix 1 that while $J_{N}^{*}(\Lambda)$ varies with both $N$ and $\underline{\Lambda}$, for sufficiently large $N, J_{N}^{*}(\Lambda)$ may be treated as if it were not dependent upon $\Lambda$. Using this result by absorbing $J_{N}^{*}(\Lambda)$ into the proportionality constant, and substituting (3.15) into ( 3.13 ), ylelds the large sample marginal posterior density for $\Lambda$,

$$
h_{\infty}^{\prime \prime}(\underline{\Lambda}) \propto \frac{\exp \left\{(-1 / 2) \operatorname{tr}\left[\underline{G}\left(\underline{\Lambda}-\underline{A}_{2}\right) \underline{v}_{2}\left(\underline{\Lambda}-\underline{A}_{2}\right) \underline{t}\right]\right\}}{\mid \underline{S}+\Psi \underline{\underline{\Lambda}})\left.\right|^{(\nu+N) / 2}} .
$$

Next note that as $N \rightarrow \infty,(\underline{S} / N)$ converges in probability to the true value $\underline{\Omega}^{\circ}$ of $\underline{\Omega}$ so that in the limit, the asymptotic posterior density of $\Lambda$ becomes concentrated on $0<\Lambda^{t}<\underline{\Omega}^{0}$. Consequently, in large samples, $h_{\infty}(\Lambda)$ may be regarded as belng concentrated on $0<\underline{\Lambda \Lambda}^{t}<(\underline{S} / N)$. That is, we take $h_{\infty}^{\prime \prime}(\underline{\Lambda})=0$ if $\left[(\underline{S} / N)-\underline{\Lambda \Lambda}^{t}\right]$ is not positive definite.

The result in (3.16) may be simplified st1ll further (In large samples) as follows. Since 


$$
\begin{aligned}
& 20 \\
& |\underline{s}+\underline{\psi}(\underline{\Lambda})|^{-(\nu+N) / 2}=\exp \left\{\left(\frac{u+N}{2}\right) \log |\underline{s}+\underline{\psi}(\underline{\Lambda})|\right\}, \\
& |\underline{s}+\underline{\psi}(\underline{\Lambda})|^{-(\nu+N) / 2} \propto \exp \left\{-\left(\frac{v+N}{2}\right) \log |\underline{I}+\underline{W}|\right\} \text {, }
\end{aligned}
$$

where $\underline{W} \equiv \underline{s}^{1 / 2} \Psi\left(\underline{\Lambda} \underline{S}^{-1 / 2}\right.$ and the proportionality constant depends only upon sample data. Note that $\underline{W}$ is a positive definite symmetric matrix whose latent roots converge in probability to zero with increasing sample size. Thus, using the argument in [8], page 277 we find that in large samples,

$$
|\underline{s}+\underline{\Psi}(\underline{\Lambda})|^{-(v+N) / 2} \propto \exp \left\{-\left(\frac{v+N}{2}\right) \operatorname{tr} \underline{s}^{-1} \underline{\psi(\Lambda)}\right\} .
$$

Combining (3.17) with (3.16) gives

$$
\left.h_{\infty}^{\prime \prime}(\underline{\Lambda}) \propto \exp \left\{(-1 / 2) \operatorname{tr}\left[\left(\underline{\Lambda}-\underline{A}_{2}\right) \underline{v}_{2}\left(\underline{\Lambda}-\underline{A}_{2}\right)^{t} \underline{G}+(\underline{\bar{s}})^{-1} \underline{\Psi} \underline{\Lambda}\right)\right]\right\},
$$

where $\underline{\bar{s}} \equiv \underline{S} /(\nu+N)$.

Now recall the matrix Identity which holds for any conformable matrices $(\underline{Q}, \underline{B}, \underline{Z})$,

$$
\operatorname{tr}\left(\underline{B Q B}^{t}\right) \underline{z}=\underline{b}^{t}(\underline{Q} \underline{Q} \underline{z} \underline{b},
$$

wher $(x)$ denotes the Kronecker product of two matrices, $B \equiv\left(b_{1}, \ldots, b_{r}\right)$ 
and $\underline{b}^{t} \equiv\left(\underline{b}_{1}^{t}, \ldots, \underline{b}_{r}^{t}\right)$. Accordingly define $\underline{A} \equiv\left(\underline{\lambda}_{2}, \ldots, \underline{\lambda}_{s}\right)$,

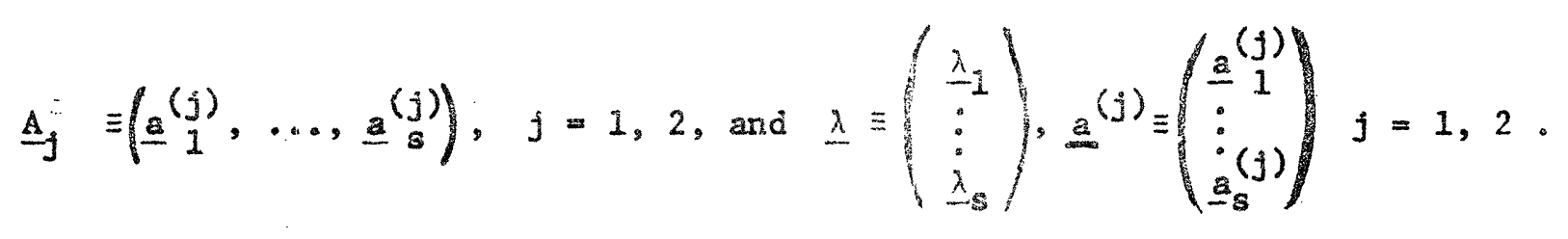

Then from $(3.4)$ and $(3.19),(3.1 .8)$ becomes

$$
\begin{aligned}
& h_{\infty}^{\prime \prime}(\underline{\Lambda}) \propto \exp \left\{( - 1 / 2 ) \left[\left(\underline{\lambda}-\underline{a}^{(2)}\right)^{t}\left(\underline{U}_{2} \propto \underline{x}\right)\left(\underline{\lambda}-\underline{a}^{(2)}\right)\right.\right. \\
& \left.\left.\left.+\left(\underline{\lambda}-\underline{a}^{(1)}\right)^{t}(\underline{v}) \underline{\underline{s}} \underline{-}^{-1}\right)\left(\underline{\alpha}-\underline{a}^{(1)}\right)\right]\right\}
\end{aligned}
$$

Combining terms and completing the square in the exponent gives the large sample marginal posterior density of the factor loading matrix

$$
\left.h_{\infty}^{\prime \prime}(\underline{\Lambda}) \propto \exp \{(-1 / 2) \underline{(\lambda-\bar{\lambda}})^{t^{t}} \underline{\Delta}^{-1}(\underline{\lambda}-\underline{\bar{\lambda}})\right\}
$$

for $(\underline{S} / N)-\underline{\Lambda}^{t}>\underline{0}$, and $h_{\infty}^{\prime \prime}(\underline{\Lambda})=0$ otherwise, where

and

$$
\underline{\Delta}^{-1} \equiv\left[\underline{v}_{1}\left(x \underline{S}^{-1}\right]+\left[\underline{v}_{2}(x),\right.\right.
$$

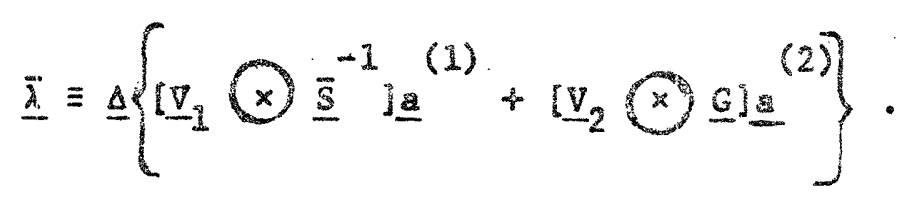


That is, the large sample marginal posterior density of $\Lambda$ is truncated normal, concentrated on $(\underline{S} / N)-\underline{\Lambda}^{t}>\underline{0}$, with modal value at $\bar{\lambda}$, defined in (3.21). Thus, if $(\underline{S} / N)-\underline{\Lambda}^{t}>\underline{0}$, a modal estimator of $\Lambda$ is $\bar{\Lambda}$, where $\underline{\bar{\Lambda}} \equiv\left(\bar{\lambda}_{1}, \ldots, \bar{\lambda}_{s}\right)$, and $\underline{\lambda}^{-t} \equiv\left(\bar{\lambda}_{1}^{t}, \ldots, \bar{\lambda}_{s}^{t}\right)$. If $(\underline{S} / \mathrm{N})-\underline{\Lambda}^{t}$ is not positive definite there is no modal estimator. In correspondence with the a priorl fudgment that the last s-p colums $\Lambda_{2}$ of $\Lambda$ are concentrated in a neighborhood of the zero matrix it may be natural in some circumstances to consider the posterfor density of $\Lambda_{1}$ given $\underline{\Lambda}_{2}=\underline{0}$ in place of that of $\underline{\Lambda}$. If the hyperparameters $\underline{a}^{(1)}$ and $\underline{a}^{(2)}$

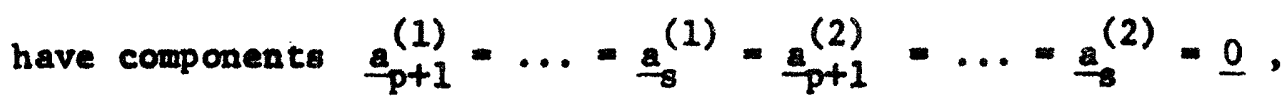
then $\frac{\lambda}{p+1}_{p}=\ldots=\bar{\lambda}_{8}=\underline{0}$, and the conditional posterior density corresponding to $(3.21) 18$

$$
h_{\infty}^{\prime \prime}\left(\underline{\Lambda}_{1} \mid \underline{\Lambda}_{2}=\underline{0}\right) \propto \exp \left\{(-1 / 2)\left({ }_{1} \underline{\lambda}-{ }_{1}{ }^{\bar{\lambda}}\right)^{t} \underline{\Delta}_{11,2}^{-1}\left(1-\lambda-{ }_{1} \underline{\lambda}\right)\right\}
$$

where ${ }_{1} \underline{\lambda}^{t}=\left(\underline{\lambda}_{1}^{t}, \ldots, \underline{\lambda}_{p}^{t}\right)$ and ${ }_{1} \underline{\lambda}^{t}=\left(\underline{\lambda}_{1}^{t}, \ldots, \underline{\lambda}_{p}^{t}\right) ;$ here $\Delta_{11.2}$ $-\Delta_{11}-\underline{\Delta}_{12} \underline{\Delta}_{22}^{-1} \underline{\Delta}_{21}$ and $\underline{\Delta}$ has been partitioned conformably with $\underline{\lambda}$. This conditional density is also truncated normal, but concentrated on $\frac{1}{N} \underline{S}-\underline{\Lambda}_{1} \underline{\Lambda}_{1}^{t}>\underline{0} \quad\left(\right.$ In place of $\frac{1}{N} \underline{S}-\underline{\Lambda \Lambda}^{t}>\underline{0}$ ). 


\section{Assessment of the Prior Distribution}

Numerical evaluation of the modal Bayes estimator, given in (3.22), depends upon knowledge of the hyperparameters, $\left\{\underline{A}_{1}, \underline{A}_{2}, \underline{V}_{1}, \underline{V}_{2}, \underline{S}_{0}, \underline{G}, v\right\}$. In this section we show that under certain conditions the hyperparamaters can be assessed in a straightforward way.

Substituting (3.12) Into (3.6) yields the joint prior density (3.3),

$$
h^{\prime}(\underline{\Lambda}, \underline{\Omega}) \propto \frac{\exp \left\{(-1 / 2) \operatorname{tr}\left[\underline{\Omega}^{-1} \Psi(\underline{\Lambda})+\Phi(\underline{\Lambda})\right]\right\}}{|\underline{\Omega}|^{(v+m+1) / 2}},
$$

for $\underline{\Omega}-\underline{\Lambda}^{t}>\underline{0}$, and $h^{\prime}(\underline{\Lambda}, \underline{\Omega})=0$ otherwise, where

$\Phi\left(\underline{\Lambda} \equiv \underline{G}\left(\underline{\Lambda}-\underline{A}_{2}\right) \underline{V}_{2}\left(\underline{\Lambda}-\underline{A}_{2}\right)^{t}\right.$. For some problems it is reasonable to take $\underline{G}=\underline{I}$. In others, having $\underline{G}$ avallable for free cholce setves as an additional degree of freedom for imposing ose's subjective bellefs on the problem and will be welcomed by many asseasors.

The marginal prior density of $\Lambda$ 1s given in (3.12). Using the asymptotic result in (3.15) shows that if $v$ is large and if $s_{0}$ is large In such a way that $\left(\underline{S}_{0} / v\right)$ remalns large, but finite, $h_{2}^{\prime}(A)$ may be written approximately as

$$
h_{\infty}^{\prime}(\Lambda) \propto \frac{\exp \{(-1 / 2) t r \underline{(\Lambda)}\}}{|\underline{\psi}(\underline{\Lambda})|^{v / 2}} .
$$


Now applying to $\underline{S}_{0}$ the same argument used in Section 3.3 for $\underline{S}$, shows that for large $v$ and $\underline{s}_{0}$,

$$
|\underline{\Psi}(\underline{\Lambda})|^{-v / 2} \propto \exp \left\{(-v / 2) \operatorname{tr} \underline{s}_{0}^{-1}\left[\left(\underline{\Lambda}-\underline{A}_{1}\right) \underline{v}_{-1}\left(\Lambda-\underline{A}_{1}\right)^{t}\right]\right\}
$$

Thus, for large $v$ and $\underline{S}_{0}$, it is approximately true that

$h_{\infty}^{\prime}(\Lambda) \propto \exp \left\{(-1 / 2) \operatorname{tr}\left[\left(\underline{\Lambda}-\underline{A}_{2}\right) \underline{V}_{2}\left(\underline{\Lambda}-\underline{A}_{2}\right)^{t} \underline{\underline{Q}}+\left(\underline{S}_{0}\right)^{-1}\left(\underline{\Lambda}-\underline{A}_{1}\right) \underline{V}_{1}\left(\underline{\Lambda}-\underline{A}_{1}\right)^{t}\right]\right\}$,

where $\underline{\mathrm{s}}_{0} \equiv\left(\underline{s}_{0} / \nu\right)$

Adapting the result in (3.19) gives

$$
\begin{aligned}
h_{\infty}^{\prime}(\Lambda) & \propto \exp \left\{( - 1 / 2 ) \left[\left(\underline{\lambda}-\underline{a}^{(2)}\right)^{t}\left(\underline{v}_{2} \otimes \underline{G}\right)\left(\underline{\lambda}-\underline{a}^{(2)}\right)\right.\right. \\
& \left.\left.+\left(\underline{\lambda}-\underline{a}^{(1)}\right)^{t} \cdot \dot{v}_{1}\left(\underline{x} \overline{\underline{s}}_{0}^{-1}\right)\left(\underline{\lambda}-\underline{a}^{(1)}\right)\right]\right\} .
\end{aligned}
$$

Combining terms and completing the square in the exponent gives the approximate marginal prior density

$$
h_{\infty}^{\prime}(\underline{\Lambda}) \propto \exp \left\{(-1 / 2)\left(\underline{\lambda}-\underline{\lambda}^{*}\right)^{t} \underline{\Delta}^{*}-1\left(\underline{\lambda}-\underline{\lambda}^{*}\right)\right\},
$$

where

$$
\underline{\Delta}^{*-1} \equiv\left[\underline{v}_{1}\left(\bar{s}_{0}^{-1}\right]+\left[\underline{v}_{2}(\underline{G}]\right.\right.
$$


and

$$
\underline{\lambda}^{*}=\underline{\Delta}^{*}\left\{\left[v_{1} \bigotimes \underline{\bar{s}}_{0}^{-1}\right] \underline{a}^{(1)}+\left[\underline{v}_{2} \bigotimes \underline{G}\right] \underline{a}^{(2)}\right\}
$$

That is, it is approximately true (for large $\nu$ and large $\underline{s}_{0}$ )

that a priori,

$$
\mathcal{L}(\underline{\lambda})=N\left(\underline{\lambda}^{*}, \underline{\Delta}^{*}\right)
$$

In order to concentrate the last s-p coluwns, $\Lambda_{2}$, of $\Lambda$ in a nelghborhood of the zero matr1x as suggested at the outset, set $\frac{a}{p+1}=\ldots$ $a_{s}^{(j)}=\underline{0}$ for $j=1,2$ and partitioning

$$
\underline{v}_{j}=\left[\begin{array}{ll}
\underline{v}_{11}^{(j)} & \underline{v}_{12}^{(j)} \\
\underline{v}_{21}^{(j)} & \underline{v}_{22}^{(j)}
\end{array}\right], \underline{v}_{22}^{(j)}:(s-p) \times(s-p),
$$

set $\underline{v}_{22}^{(j)}=t I, t$ arbitrarily large. The corresponding variance of $\underline{\Lambda}_{2}$ w11l then be small. That 1s, 1 is approximately true that a ptior1, $\Lambda_{2}$ is normally distributed with mean zero and a covariance matrix whose largest latent root 18 approximately zero. 
Next concentrate on $\underline{\Omega} \mid \underline{\Lambda}$. By substituting (3.5) into (3.4) we find that for $\underline{\Omega}-\underline{\Lambda \Lambda}^{t}>\underline{0}$,

$$
h_{1}^{\prime}(\Omega \mid \Lambda)=\frac{\left|\Lambda \Lambda^{t}\right|^{\nu / 2} \exp \left\{(-1 / 2) \operatorname{tr}\left[\underline{\Omega}^{-1} \psi(\Lambda)\right]\right\}}{|\Omega|^{(\nu+m+1) / 2}{ }_{1}{ }_{1}\left(\frac{\nu}{2} ; \frac{\nu+m+1}{2} ;(-1 / 2)(\Lambda \Lambda)^{t} \psi(\Lambda)\right)} .
$$

Now assume $\underline{S}_{0}$ and $\nu$ are large. Adapting the result in (3.15) to the ${ }_{1} F_{1}$ function in the last equation gives approximately,

$$
h_{1}^{\prime}(\underline{\Omega} \mid \underline{\Lambda}) \propto \frac{|\underline{\underline{\Lambda}}(\underline{\Lambda})|^{\nu / 2} \exp \left\{(-1 / 2) \operatorname{tr}\left[\underline{\Omega}^{-1} \psi(\Lambda)\right]\right\}}{|\underline{\Omega}|^{(\nu+1) / 2}},
$$

for $\underline{\Omega}-\underline{\Lambda}^{t}>\underline{0}$, and $h_{1}^{\prime}(\underline{\Omega} \mid \underline{\Lambda})=0$ otherw1se. But by taking $\underline{s}_{0}$ and $v$ large so that $\bar{s}_{0}$ is also large, most of the mass of the distribution 
of $\underline{\Omega} \mid \underline{\Lambda}$. Is moved far from zero so that $P\left\{\underline{\Omega}-\underline{\Lambda}^{t}>\underline{0} \underline{\Lambda}\right\}$ is close to unity. Therefore, we assume as an approximation, that (4.3) holds for all $\underline{\Omega}>\underline{0}$, so that a prior1, $\underline{1}$ follows an inverted Wishart distribution.

Taking moments in $(4.2)$ and (4.3) shows that a priori, we have approximately (cf. [6])

$$
\begin{gathered}
E(\underline{\lambda})=\underline{\lambda}^{*}, \operatorname{var}(\underline{\lambda})=\underline{\Delta}^{*}, \\
E(\underline{\Omega} \mid \underline{\Lambda})=\frac{\left.\underline{S}_{0}+\underline{(\Lambda}-\underline{A}_{1}\right) \underline{v}_{1}\left(\underline{\Lambda}-\underline{A}_{1}\right)}{\nu-m-1}, v>m+1 \\
\operatorname{var}\left(\omega_{11} \mid \underline{\Lambda}\right)=\frac{2 \psi_{1 i}^{2}}{(\nu-m-1)^{2}(\nu-m-3)}, v>m+3,
\end{gathered}
$$

and for $1 \neq i$,

$$
\operatorname{var}\left(w_{i j} \mid \underline{1}\right)=\frac{\left[\psi_{i 1} \psi_{j j}+\frac{v-m+1}{v-m-1} \psi_{1 j}\right]}{(v-m)(v-m-1)(v-m-3)},
$$

for $v>m+3$, and

$$
\operatorname{cov}\left[\left(\omega_{i f}, \omega_{k l}\right) \mid \underline{\Lambda}\right]=\frac{\left[\frac{2}{\nu-m-1} \psi_{1 j} \psi_{k \ell}+\psi_{1 j} \psi_{j l}+\psi_{i \ell} \psi_{k j}\right]}{(\nu-m)(\nu-m-1)(\nu-m-3)},
$$


for $v>m+3$, where $\Omega \equiv\left(\omega_{1 j}\right)$, and $\psi_{1 j}$ denotes the $(1, j)$ element of $\psi(\underline{\Lambda})$.

In some situations there is some underlying theory to suggest values for the first two moments of $\Lambda$ and $\underline{\Omega} \mid \underline{\Lambda}$. In other situations one or several factor analyses may have been carried out on earlier data under similar circumstances. In such an event, this earlier data can be used to fit the moments of $\Lambda$ and $\underline{\Omega} \underline{\Lambda}$. In any case, the hyperparameters may then be found by solving the moment equations (4.4) - (4.8) simultaneously, adjusting $\nu$ and $\underline{s}_{0}$ so that they are large enough to provide any preassigned level of comfort to the analyst that his approximations are reasonably valid. For example, suppose we take $\left[\underline{s}_{0} /(\nu-m-1)\right] \equiv \sigma^{2} \underline{I}$, for some $\sigma^{2}$ to be determined. From (4.5) it is seen that this can be made to be the dominant term in $E(\underline{\Omega} \mid \underline{\Lambda})$. If we wish to ensure that $\left[\frac{\underline{s}_{0}}{\nu-m-1}-\underline{\Lambda \Lambda}\right]>\underline{0}$, for given $\underline{\Lambda}$, we must choose $\sigma^{2}$ so that for any $\underline{z} \neq \underline{0}, \sigma^{2} \underline{z}^{t} \underline{z}>\underline{z}^{t} \underline{\Lambda \Lambda}^{t} \underline{z}$. But $1 t$ is well known that $\left(\underline{z}^{t} \underline{\Lambda \Lambda}^{t} \underline{z}\right) /\left(\underline{z}^{t} \underline{z}\right)$ never exceeds $\phi_{M}$, the largest latent root of $\underline{\Lambda}^{t}$. So we only need to choose $\sigma^{2}$ larger than $\phi_{M}$, for given $\Lambda$, to satisfy the positive definiteness condition for the mean of $\underline{\Omega} \mid \underline{\Lambda}$. Then, choosing $\sigma^{2}$ even larger provides an increasingly closer approximation to the theoretical prior distribution. 
It may be noted that since $\underline{\Omega}=\underline{\Lambda \Lambda}^{t}+\underline{\Sigma}, E(\underline{\Omega} \mid \underline{\Lambda})=\underline{\Lambda}^{t}+E(\underline{\Sigma})$, and $\operatorname{var}(\underline{\Omega} \mid \underline{\Lambda})=\operatorname{var}(\underline{\Sigma})$. Thus, the task of assessing hyperparameters may be carried out by thinking about assessing moments of $\underline{\Sigma}$. This is quite analogous to the classical factor analysis problem of estimating communalities.

Suppose the results of an earller factor analysis were avallable and $(\underline{\Lambda}, \underline{\Sigma})$ were estimated by the analysis as $(\underline{\tilde{\Lambda}}, \underline{\Sigma})$. A reasonable assessment might then be

$$
E(\underline{\Omega} \mid \underline{\Lambda})=\tilde{\Lambda}^{t}+\tilde{\Sigma}=\tilde{\Omega} \text {. }
$$

Finally, note from (4.3), and (4.5) that fixing $\tilde{\Omega}$ and $v$ is sufficlent to define the entire prior distribution of $(\Omega \mid \Lambda$ ) (cf. [8], page 112, Remark). That is, when $\underline{\Omega}$ and $v$ are given, not only is the left hand side of (4.5) known, but also, the left hand sldes of (4.6) - (4.8). 


\section{Append1x 1}

In this appendix we show that $\mathrm{J}_{\mathrm{N}}^{*}(\Lambda)$, defined in (3.14), varles with both $N$ and $\Lambda$, but for large $N$, the dependence of $J_{N}^{*}(\Lambda)$ on $\Lambda$ disappears.

Suppose $\underline{P}: m \times m$ is a positive definite symetric random matrix with density

$$
f(\underline{P})=k(m, N)|\underline{\underline{p}}|^{\frac{\nu+N-m-1}{2}} \exp \{-t r \underline{\underline{p}}\}, \underline{\underline{P}}>\underline{0}
$$

and $f(\underline{P})=0$ otherwise, where $\nu+N \geqslant m$, and

$$
[k(m, N)]^{-1}=\pi^{m(m-1) / 4} \prod_{j=1}^{m} \Gamma\left(\frac{j+N+1-j}{2}\right) .
$$

Define

$$
J_{N}(\underline{\Lambda}) \equiv \int_{\underline{K}_{N}-\underline{P}>0} f(\underline{P}) d \underline{P},
$$

where

$$
\underline{K}_{N} \equiv(1 / 2)[\underline{\psi(\Lambda)}+\underline{s}]^{1 / 2}\left(\underline{\Lambda \Lambda^{t}}\right)^{-1}[\underline{\Psi}(\underline{\Lambda})+\underline{s}]^{1 / 2},
$$

and $\underline{\Psi}(\underline{\Lambda})$ denotes the positive definite symmetric quadratic form 
$\Psi(\underline{\Lambda}) \equiv \underline{S}_{0}+\left(\underline{\Lambda}-\underline{A}_{1}\right) \underline{v}_{1}\left(\underline{\Lambda}-\underline{A}_{1}\right)^{t}$ Introduced In (3.4). Note from (3.14) that $J_{N}(\underline{\Lambda})=k(m, N) J_{N}^{*}(\underline{\Lambda})$. We now show that for large $N, J_{N}(\underline{\Lambda})$ does not depend upon $\Lambda$, therefore, neither does $J_{N}^{*}(\Lambda)$.

Theorem Al: $\operatorname{Lim}_{\mathrm{N} \rightarrow \infty} \mathrm{J}_{\mathrm{N}}(\underline{\Lambda})=1$.

Proof: $\quad$ Since $\underline{P}$ follows a Wishart distribution with scale matrix $I / 2, \quad E(\underline{P})=[(\nu+N) / 2] \underline{I}$. By the strong law of large numbers ${ }^{1}$ (SLLN.)

$$
\lim _{N \rightarrow \infty}\left(\frac{2 P}{N+v}\right)=\lim _{N \rightarrow \infty}\left(\frac{2 P}{N}\right)=I, \text { a.s., }
$$

and $\lim (\underline{s} / \mathrm{N})=\underline{\Omega}$, a.s.

$$
\text { If } \underline{R} \equiv \underline{\Omega}^{1 / 2}\left(\underline{\Lambda \Lambda}^{t}\right)^{-1} \underline{\Omega}^{1 / 2} \text {, by the SLLN., } \lim _{N \rightarrow \infty}\left(\frac{2 \mathrm{~K}}{N}\right)=\underline{R} \text {, a.s., }
$$

(because $\underline{\psi(\Lambda)}$ does not depend upon $N, \underline{\Psi(\Lambda) / N}$ converges to zero). Thus,

$$
\lim _{N \rightarrow \infty}\left(\frac{2 K_{N}}{N}-\frac{2 \underline{P}}{N}\right)=\underline{R}-\underline{I}, \text { a.s. }
$$

Next note that $\underline{R}=\underline{\Omega}^{1 / 2}(\underline{\Omega}-\underline{\Sigma})^{-1} \underline{\Omega}^{1 / 2}=\left[\underline{I}-\underline{\Omega}^{-1 / 2} \underline{\Sigma \Omega}^{-1 / 2}\right]^{-1}$,

and since $\underline{\Omega}-\underline{\Sigma}>\underline{0}, \underline{I}-\underline{\underline{S}}^{-1 / 2} \underline{\Sigma S t}^{-1 / 2}>\underline{Q}$. Therefore, if $(1-\theta)$ denotes a latent root of $I-\underline{\Omega}^{-1 / 2} \underline{\Omega}^{-1 / 2},(1-\theta)>0$. Moreover, since

1 Convergence almost surely is denoted by a.s. 
$\theta$ is a latent root of $\Omega^{-1 / 2} \underline{\Sigma}^{-1 / 2}$, and the latter is positive definite, we must have $0<\theta<1$. Thus, $\underline{R}$ may be expanded in a power series of the form

$$
\underline{R}=\sum_{j=0}^{\infty}\left(\underline{\Omega}^{-1 / 2} \underline{\Sigma \Omega^{-1 / 2}}\right)^{j},
$$

so that if $\underline{G} \equiv \sum_{1}^{\infty}\left(\underline{\Omega}^{-1 / 2} \underline{\Sigma \Omega}-1 / 2\right)^{j}$, where clearly $\underline{G}>\underline{0}, \underline{R}=\underline{I}+\underline{G}$.

Therefore from (A.1)

$$
\lim _{N \rightarrow \infty} \frac{2 K}{N}-\frac{2 P}{N}=G>\underline{0} \text {, a.s. }
$$

Equivalently,

$$
\lim _{N \rightarrow \infty} P\left\{\frac{2}{N}\left(\underline{K}_{N}-\underline{P}\right)>\underline{0}\right\}=1 \text {. }
$$

Since by definition,

$$
J_{N}(\underline{\Lambda})=P\left\{\underline{K}_{N}-\underline{P}>\underline{0}\right\}=P\left\{\frac{2}{N}\left(\underline{R}_{N}-\underline{P}\right)>\underline{0}\right\},
$$

the theorem follows.

Thus, for large $N, J_{N}^{*}(\Lambda)$ depends only upon $(m, N)$ and not on $\Lambda$. 


\section{References}

[ 1] Dreze, J. H. (1968) "L1mited Information Estimation from a Bayesian Vlewpoint," Report No. 6816, Center for Operations Research and Econometrics, Catholic University of Louvaln, Heverlee, Belgium.

[ 2] F1sher, F. (1968) The Ident1fication Problem in Econometr1c8, New York: McGraw H111, Inc.

[ 3] Herz, C. S. (1955) "Bessel Functions of Matrix Argument", Annals of Mathematics, Vol. 61, No. 3, pp. 474-523.

[ 4] James, A. (1964) "Distribution of Matrix Varlates and Latent Roots Derived from Normal Samples", Annals of Math. Stat., Vol. 35, pp. 475-501.

[ t? Jeffreys, H. (1961) Theory of Probab1115y, Oxford: Clarendon Press.

[ 6] Kaufman, G. M. (1967) "Some Bayesian Moment Formulae", Report No. 6710, Center for Operations Research and Econometrics, Catholic University of Louvaln, Heverlee, Belgium.

[ 7] Mulaik, S. A. (1972) The Foundations of Factor Analys is, New York: MeGraw-H111 Book Co.

[ 8] Press, S. J. (1972) Applied Multivarlate Analysis; New York: Holt, Rinehart and Winston, Inc. 